\title{
OSTEOID OSTEOMA WITH UNUSUAL SYMPTOMS
}

\author{
Case Report \\ O. J. Vaughan-Jackson, London, Exglaxd \\ From the Orthopaedic Department, St Bartholomew's Hospital, Rochester, Lent
}

Osteoid osteoma has come to be associated with a typical clinical history. There is gradual, unexplained onset of pain, which is described as "boring" and " aching," and occurs especially at night; it is relieved by aspirin, though often by nothing else. In most of the recorded cases the pain has been closely referred to the site of the osteoid osteoma. The case presented here is of interest in that the distribution of the pain was misleading, and suggests that in cases in which the pain is typical, but the radiographs show no abnormality in the area complained of, a wider radiological search for the lesion may be adrisable.

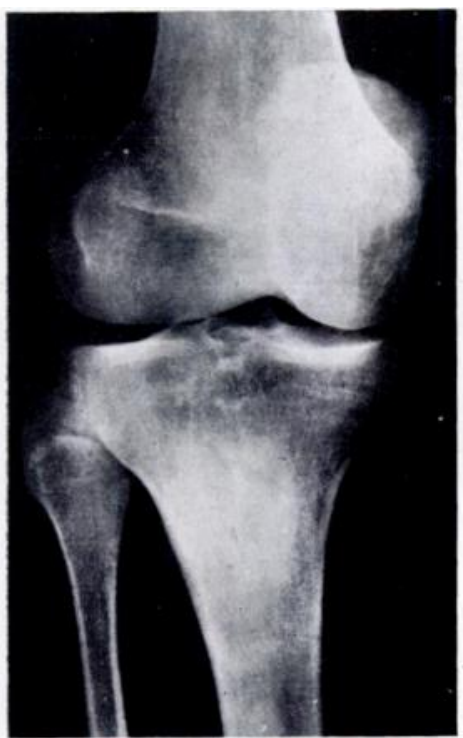

FIG. 1

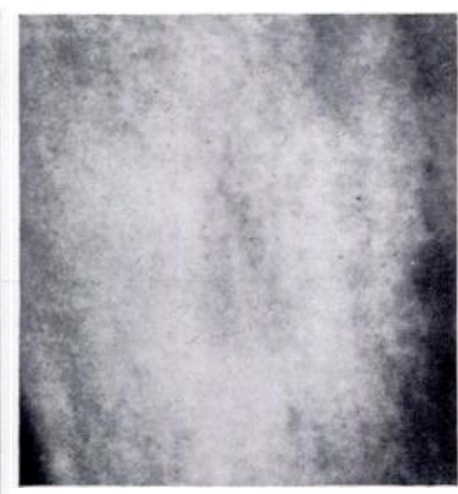

FIG. 2

Figures 1 and 3 -Radiographs of the lesion; Figure 2-An enlargement of the central area. enlargement of the central area.
Note the "sequestrum " within the translucent zone.

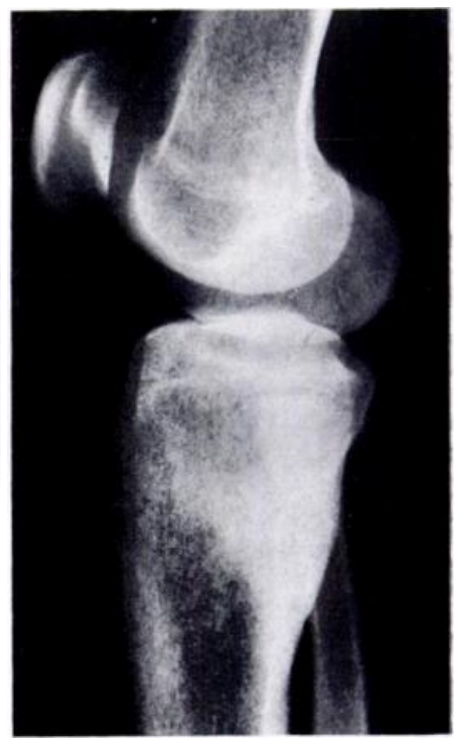

FIG. 3

R. V. H., aged twenty-two years, a travelling salesman, first attended St Bartholomew's Hospital, Rochester, in January 1949. He stated that two and a half years previously, while serving in the Army in the Middle East, he began to feel pain deep in the substance of the right thigh; very occasionally the pain spread into the right knee. From that time onwards he was never free from pain. He described its character as very wearing and boring, like toothache. He found that it was always relieved by aspirin, which he took regularly many times a day. The pain woke him at night about 2 a.m. and 5 a.m. There were no other symptoms. Soon after the onset of the pain he had been investigated by Army medical officers who failed to find any organic disease. He was seen by several psychiatrists who were in agreement that he was of a mentally immature and slightly unstable type. It was concluded that the pain was functional in origin, and he was ultimately invalided from the Army on those grounds.

On examination, there were no abnormal physical signs except slight wasting of the right 
thigh. Radiographs of the hip and femur showed no abnormality. The patient appeared rather anxious and nervous, and did not make a very favourable impression except in being a good and consistent witness. For this reason the case was several times thoroughly reviewed. Eventually, because of the complaint of occasional pain in the knee, the joint was radiographed. No abnormality was seen in the knee itself, but in one film there appeared to be a little density in the centre of the shaft of the tibia. A further radiograph, centred on this area, was made. It showed a well-marked area of sclerosis in the posterior part of the upper end of the shaft of the tibia (Figs. 1 and 3). In the centre of this area there was an ill-defined lesion which had the appearance of a small oval cavity with a doubtful small sequestrum at its centre (Fig. 2 ). The radiographs suggested a chronic abscess of the tibia, but in view of the nocturnal pain the diagnosis of osteoid osteoma was regarded as a distinct possibility, and the patient was advised to submit to a biopsy of this abnormal area. Operation was undertaken through a posterior approach reflecting the popliteus. The raised irregular sclerosed bone on the posterior face of the tibia was removed with a gouge and the plane of cleavage fortunately passed through the centre of the lesion. It was revealed as a small purplish oval mass of what appeared to be cancellous bone, one centimetre in length, lying in pale sclerosed bone. The mass had adhered to the fragment removed, and left behind it

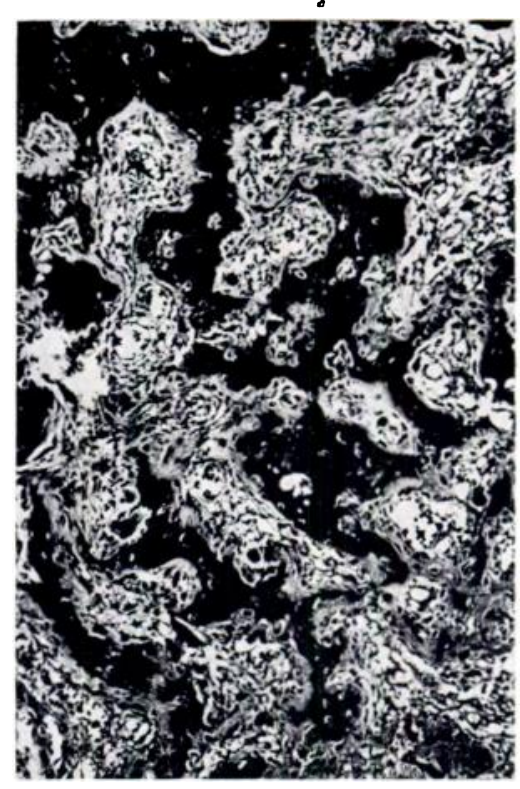

Fig. 4

Photomicrograph of the tumour, showing deeply stained trabeculae of osseous tissue covered by deep osteoid seams $(\because 4 \overline{5})$.

in the tibia a perfectly smooth oval bed. There was no pus or granulation tissue, and nothing to suggest an inflammatory lesion. The wound was closed.

The operation gave immediate relief from pain, and the patient was free from symptoms ten months later. The report on the pathological section was "osteoid osteoma from tibia" (Fig. 4).

I wish to express my thanks to Professor Dorothy Russell for the pathological examination of the specimen and for the photomicrograph. 